Mol. Cryst. Liq. Cryst., 1988, Vol. 155, pp. 495-500

Photocopying permitted by license only

(C) 1988 Gordon and Breach Science Publishers S.A.

Printed in the United States of America

\title{
A NEW HARD-PARTICLE MODEL FOR ANISOTROPIC FLUIDS
}

\section{M.C. DURO AND J.A. MARTIN-PEREDA}

E.T.S.I. Telecomunicacion, U.P.M., Madrid 28040, Spain

Abstract We report a new hard-particle model system consisting of hard cylinders. we have determined the geometrical conditions that let us know whether or not two given cylinders overlap. In addition we have carried out Monte Carlo simulations sampling the canonical ensemble on this system. the numerical results indicate that this system exhibits mesomorphic behaviour.

\section{INTRODUCTION}

Computer simulations on models of hard particles have pointed out that the stable phases exhibited by such a system depend in a rather intricate way on the shape of the constituent particles 1 . For instance, hard spheres do not form anisotropic phases, hard ellipsoid exhibit a nematic phase and hard parallel spherocylinders show smectic behaviour. At present our knowledge of systems of nonspherical particles is very limited and very much effort is needed to find out whether these models can be a useful reference for real liquids crystals. In this connection, previous studies pointed out that great numerical difficulties can arise whenever one deals with simulations of systems of hard particles if these systems have densities and particle size in the range of liquid crystals ${ }^{2-3}$ because such simulations are very time-consuming. Recently we performed an exhaustive Monte Carlo simulation on a system of hard cylinders with length-to-width ratio $\mathrm{L} / \mathrm{D}=4.44$ and packing fraction $\eta=0.646$ (these values were calculated from data published for a typical nematic system: MBBA). In this study we showed that in such conditions the hard cylinder system exhibit a nematic phase ${ }^{4}$. 


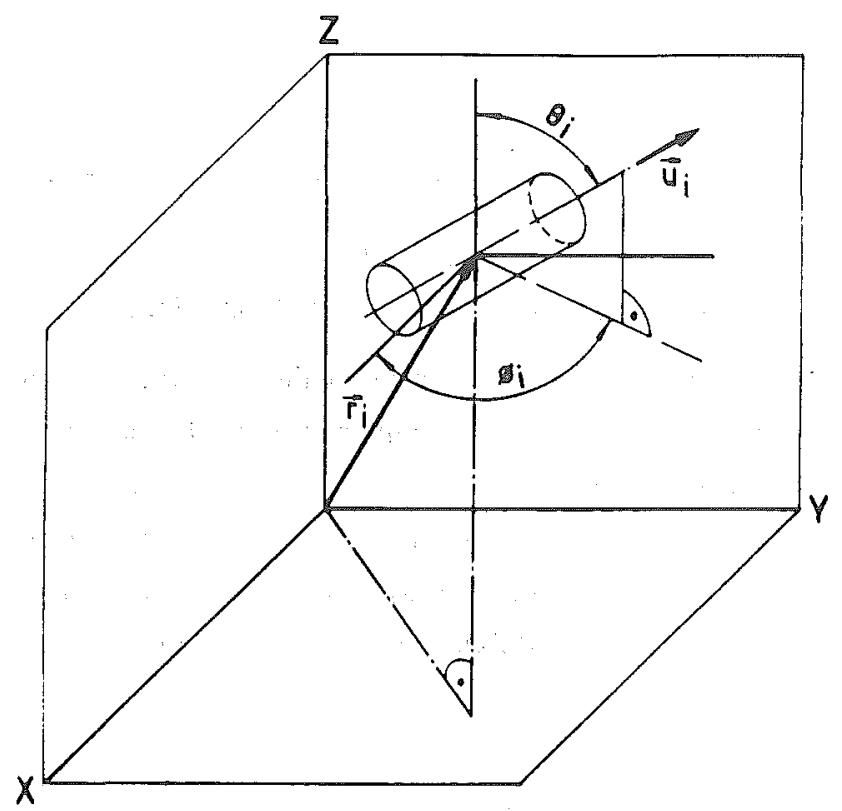

FIGURE 1 . The position and orientation of each cylinder is specified by $\underline{r}_{i}$ and $\underline{\underline{u}}_{i}$ (or the polar angles $\Theta_{i}, \phi_{i}$ ).

The present paper describes the hard-cylinder potential together with a computer study of this system. The length-to-width ratio considered is $\mathrm{L} / \mathrm{D}=4.44$. We shall present new results on the structure of this model when the packing fraction varies from 0.646 to 0.394 .

\section{HARD CYLINDER POTENTIAL}

Let us considered a system composed of $\mathrm{N}$ hard cylinders in a volume $\mathrm{V}$. The length of a cylinder is $\mathrm{L}$ and its diameter is $\mathrm{D}$. The position of each cylinder inside the system is specified in the usual way by the center of mass position vector $\underline{\underline{r}}$, and a unit vector parallel to the cylinder long axis $\underline{\mathbf{u}}_{\mathbf{i}}$ (see Fig. 1). From these data $\left(\underline{\mathbf{r}}_{\mathbf{i}}, \underline{\mathrm{u}}_{\mathbf{i}}, \mathrm{L}\right.$ and $\left.\mathrm{D}\right)$ we developed the geometrical criterion that lets us know whether or not two given cylinders overlap. As the test whether two particles intersect is the most frequent operation in the $\mathrm{MC}$ program it is worthwhile to make this computation 

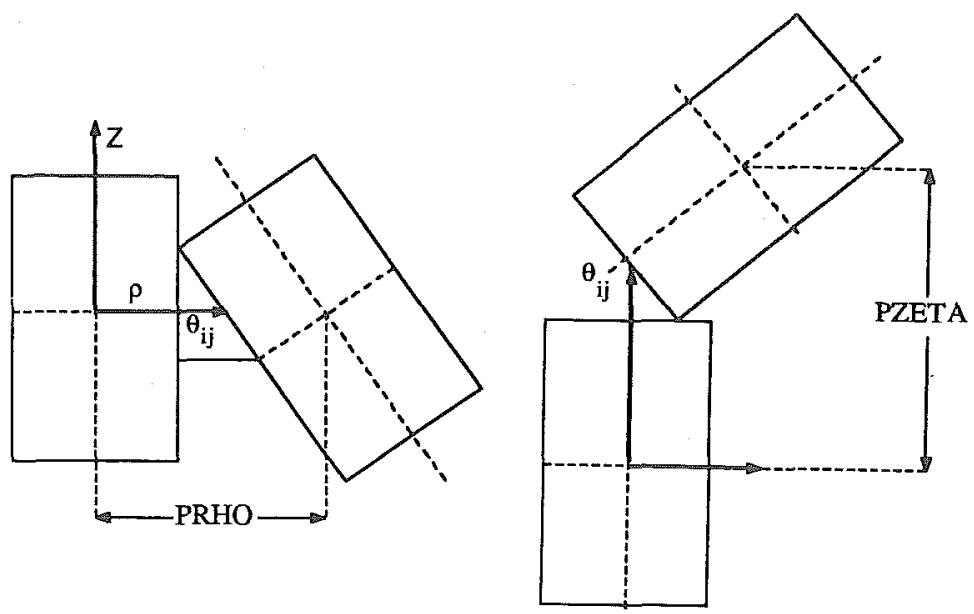

FIGURE 2 Distances of closest approach as used in the overlap criterion.

cheap. To do that we proceed in four steps:

1 - We test if the distance $\left|\underline{r}_{\mathrm{j} j}\right|$ between particles $\mathrm{i}$ and $\mathrm{j}$ is more or less than than $\mathrm{DCC}\left(\mathrm{DCC}=\left(\mathrm{L}^{2}+\mathrm{D}^{2}\right)^{1 / 2}\right)$

2-If it is less than DCC we test if the distance between the long axes is more or less than D. If this distance is less than D it is necessary to continue. Then we calculate the values of $\left|z_{i j}\right|$ and $\rho_{i j}$ in the proper frame (notice that $\mathrm{r}_{\mathrm{ij}}{ }^{2}=\mathrm{z}_{\mathrm{ij}}{ }^{2}+\rho_{\mathrm{ij}}{ }^{2}$ ).

3- If the cylinders are parallel we test if $\left|z_{i j}\right|$ is less than $L$ and if $\rho_{i j}$ is less than $\mathrm{D}$ (if both conditions hold the cylinders overlap).

4- if the cylinders are not parallel the test is similar to the previous case but now the distances of closest approach are PRHO and PZETA (see Fig.2).

\section{COMPUTER SIMULATIONS}

The simulations described in the present paper were performed using the MC method sampling the canonical ensemble. The simulation box was selected cubic and the sample size $N=150$. Markov's chain was 
generated in the usual way for rigid particles, i.e. we generated a succession of trial moves in which we attempted to change the coordinates of individual cylinders (a particle move consisted of a combined translation and reorientation). The allowed configurations were selected with the use of the nonoverlapping criterion defined above.

The high-density considered by us was $\eta=0.646$, in this run our interest was focused in three quantities closely connected with structural properties of the system: pair radial distribution function $\mathrm{g}(\mathrm{r})$, structure factor $\mathrm{S}(\underline{\mathrm{k}})$ and orientational order-parameter as defined Vieillard-Baron 5 :

$$
M=1 / N^{2} \sum_{i=1}^{N} \sum_{j=1}^{N} \cos 2 \theta_{i j},
$$

where $\theta_{\mathrm{ij}}$ is the angle between the axes of two given cylinders. These quantities were used to evaluate the stochastic evolution of the system structure along the run and characterize the stable phase. The obtained results of this run have been reported in Ref. 4, they led to conclude that in such conditions the system exhibits a nematic phase practically oriented. From this fact we concluded that it will be interesting to study the density dependence of the pair distribution function and the order parameter. The duration of the run for $\eta=0.646$ starting from a tetragonal lattice was 12 million particle moves and our MC program ran at a speed of $1.510^{5}$ particle moves per hour of CPU time in a IBM 4341 . In order to perform the abovementioned study in a reasonable time we used the common successive expansion method 6 : subsequent runs were started from previously equilibrated configurations at slightly higher density (a number of $2.5 \times 10^{5}$ particle moves were used to obtain the new equilibrated configurations).

TABLE I order parameter $M$ as a function of the packing fraction $\eta$.
$\eta$
$\begin{array}{lll}0.590 & 0.549 & 0.511\end{array}$
0.478
0.448
0.4180 .394
M
0.995
0.992
0.991
0.988
0.983
$\begin{array}{lll}0.981 & 0.968\end{array}$ 


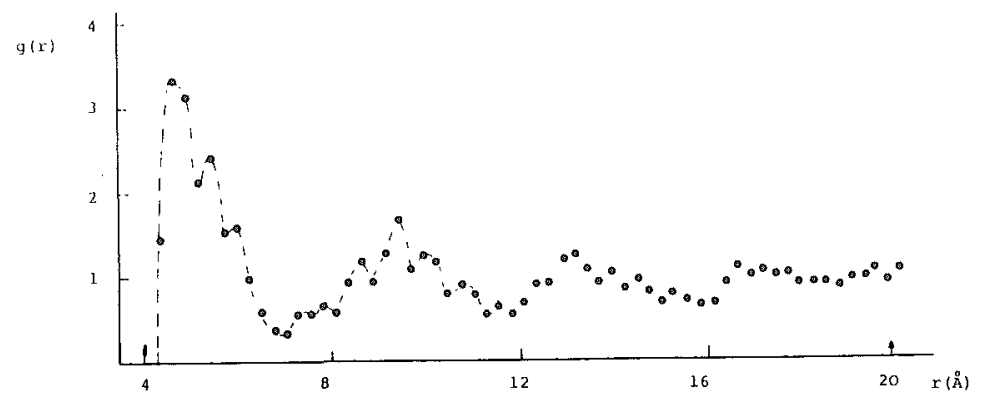

FIGURE 3. Pair radial distribution function for $\eta=0.590$

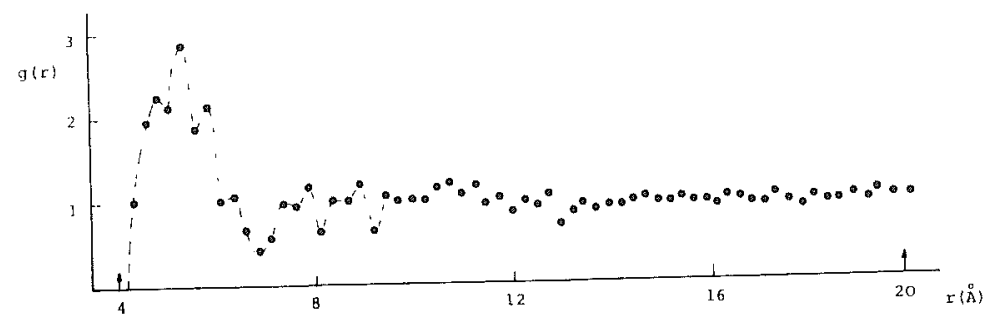

FIGURE 4. Pair radial distribution function for $\eta=0.448$

The evolution of the orientational order of the system is shown in table I. The $g(r)$ profiles for two different packings fractions are given in Figs. 3 and 4 . Finally we performed another run at the packing fraction value 0.394 starting from a tetragonal lattice, in this case after 5.2 million particle moves $M$ turned out to be 0.742 .

It is worth noting that at the low packing-fraction considered we found a great fluctuation in the value of the orientational order parameter, such fluctuation could be a consequence of the fact that at this packing fraction the system is near a phase transition. It is well known that in this critical region very large fluctuations occur and the system spends part of its time in a nematic-like and part in an isotropic-like situation. This is shown for example in the histogram of the frequency of occurrence of the second rank order parameter ${ }^{7}$. In any case we think that it would be interesting to 
perform detailed calculations in order to find the exact location of this transition point.

This research was performed under the auspices of the CAICYT, grant number $1564 / 82$.

This manuscript was written during the stay of one of us (M.C.D.) at the FOM-institute for Atomic and Molecular Physics in Amsterdam. We wish to express our gratitude to D.Frenkel, J.Veerman and N.R.Walet for their hospitality.

\section{REFERENCES}

1. D. Frenkel, Mol. Phys. 601 (1987)

2. J. Vieillard-Baron, Mol. Phys. 28809 (1974)

3. G. R. Luckhust \& G. W. Gray ed., The Molecular Physics of Liquid Crystals, (Academic London, 1979) p.197

4. M. C. Duro, J. A. Martin-Pereda \& L. M. Sese, "Monte Carlo simulation on a system of hard cylinders at very high packing fraction", Phys. Rev. A, in press

5. J. Vieillard-Baron, J. Chem Phys. 564792 (1972)

6. D. Frenkel \& B. M. Mulder, Mol. Phys. 551171 (1985)

7. P. A. Lebwohl \& G. Lasher, Phys. Rev. A6 426 (1972) 